Para enlazar con este artículo / To link to this article:

http://dx.doi.org/10.6035/MonTI.2019.11.8

Para citar este artículo / To cite this article:

Martinez, Patrick. (2019) "La traducción-vigilancia." In: Tolosa Igualada, Miguel \& Álvaro Echeverri (eds.) 2019. Porque algo tiene que cambiar. La formación de traductores e intérpretes: Presente \& futuro / Because something should change: Present \& Future Training of Translators and Interpreters. MonTI 11, pp. 215-241.

\title{
LA TRADUCCIÓN-VIGILANCIA
}

\author{
Patrick Martinez \\ patrick.martinez@ua.es \\ Universidad de Alicante
}

\section{Resumen}

El mercado de traducción y la traducción como actividad profesional evolucionan de forma constante. Aparecen nuevas solicitudes de servicios en el mercado que van más allá la traducción de un documento de una lengua a otra. Surge la necesidad de la vigilancia multilingüe: el seguimiento de información multilingüe y multisoporte encargado por empresas u organismos públicos con el fin de obtener datos en la lengua de destino para una toma de decisión óptima. El traductor se transforma en el actor principal de un proceso que implica el desarrollo de estrategias de búsqueda, la vigilancia informativa estratégica y la traducción adaptada y selectiva de contenidos para favorecer la toma de decisión del destinatario final.

\begin{abstract}
"Translation monitoring"

The translation market and translation as a professional activity are in constant evolution. New requests appear in the market, that go beyond the standard translation service from one language to another. Translators are requested by companies or public organisations to perform multilingual information monitoring, in order to obtain data or information in the target language. The translator becomes the main actor in a process involving the development of search strategies, strategic multilingual monitoring and the adapted and selective translation of information with the clear aim of facilitating the decision making of the final customer.
\end{abstract}

Palabras clave: Traducción. Vigilancia. Multilingüismo. Inteligencia económica.

Keywords: Translation. Monitoring. Multilinguism. Strategy. Business intelligence. 



\section{Introducción}

La traducción es una de las actividades profesionales que evolucionan más rápido en el marco de las profesiones de la comunicación. Esto se puede ver no solamente en las estrategias de traducción y los campos de especialización, sino también en las modalidades de traducción y contextos de comunicación. De este modo, además de la clara evolución de la actividad de traducción en sí, también se amplía el ámbito de actuación del traductor, el abanico de servicios que puede prestar a sus clientes. En este sentido, ha aparecido en los últimos años en el mercado profesional de la traducción un tipo de encargo en el que el traductor tiene que ir más allá de la 'simple' transmisión entre una lengua o cultura, y otra. Se plantea al traductor un tipo de encargo que no se centra en la necesidad de traducción de un texto concreto sino en la necesidad de acceder a una información multilingüe, multicultural y multisoporte a la cual sólo se puede llegar gracias a la traducción y, por ende, al traductor.

Una multinacional con sede en España, para la cual habíamos prestado servicios de traducción en repetidas ocasiones en el marco de nuestra actividad profesional como agencia de traducción, solicitó nuestros servicios porque le interesaba saber todo lo que se decía sobre un competidor suyo en el extranjero. El encargo del cliente difería profundamente de los que habíamos tratado hasta la fecha. En este caso concreto el cliente no nos facilitó un texto para su traducción, sino que nos planteó una solicitud precisa de información no puntual, más bien un seguimiento informativo. El cliente, sin realmente conocer el tipo de servicio que nos pidió, quería que realizáramos una vigilancia informativa multilingüe acerca de un competidor suyo en Francia. El problema de nuestro cliente residía en el hecho de que no sabía cómo o dónde buscar y monitorizar información, y tanto el idioma como el contexto sociocultural constituían obstáculos infranqueables para él. Nuestra tarea en este encargo de traducción un tanto peculiar consistió en 'vigilar' al competidor francés de nuestro cliente, realizar un seguimiento informativo, mantenernos alerta acerca de los datos que pudieran aparecer, encontrar aquella información relevante y adecuada al contenido del encargo, todo ello en soportes emitidos en lengua extranjera, para finalmente entregar al cliente información útil en su idioma. Difería 
tanto el encargo planteado de un encargo convencional de traducción, como también difería el producto final solicitado: no nos solicitaba, evidentemente, una traducción en sí, sino información traducida en su lengua después de haberla filtrado y analizado. Por otro lado, se nos indicó el objetivo claro de la información que íbamos a facilitar: la toma de decisión, la interacción con el entorno de la empresa. El informe que entregamos constituyó una pieza clave para la orientación de la estrategia de la empresa de nuestro cliente. Nos dimos cuenta de que nuestra labor, la actividad de traducción, cambiaba radicalmente. Ya no nos encontrábamos en un esquema de traducción convencional con la recepción de un texto (en el formato que sea) en una determinada lengua para que lo tradujésemos en la lengua que nos indicara el cliente, sino que tuvimos que realizar una tarea de reflexión acerca de las necesidades informativas del cliente, encontrar aquellas fuentes de información que fueran pertinentes e incluso determinar las lenguas y área geográfica en las que teníamos que realizar la búsqueda ${ }^{1}$.

Hoy en día, el desarrollo de Internet y la imparable globalización han provocado un cambio de relación con la información. Esta se ha transformado en materia prima de alto valor tanto para empresas como organismos públicos ya que el acceso a la misma, su control, gestión, análisis e interpretación pueden constituir una gran ventaja competitiva para el que la posea. El problema no es la falta de información sino más bien lo contrario, la auténtica avalancha que ha provocado el aumento de velocidad de transferencia de datos y la generalización del uso de Internet tanto a nivel profesional como personal. Para ilustrar la ingente cantidad de datos generados podríamos fijarnos en lo que ocurre cada minuto en Internet: se publican 571 páginas webs, se mandan más de 156 millones de emails, los usuarios publican más de 400 horas de video en Youtube, el comercio en línea genera más de 270.000 dólares (Moreno 2018). En definitiva, en Internet podemos encontrar prácticamente todo lo que queremos saber sobre cualquier cosa, si sabemos buscar de forma adecuada y eficiente. La paradoja estriba en el hecho de que cuanta más información es generada y cuanto más rápida es la velocidad de circulación de la misma, más difícil es encontrar aquel dato, aquella información que realmente necesitamos y que podría transformarse en un alto valor añadido.

Por lo tanto, es necesario hallar dicha información, tratarla y analizarla. En este sentido, la 'vigilancia' desempeña un papel fundamental. La vigilancia es la actividad de seguimiento informativo continuo sobre una temática concreta

1. En este caso, el competidor de nuestro cliente tenía actividades en el territorio francés pero también en Bélgica y otros países europeos. 
con el fin de encontrar información pertinente y relevante. La vigilancia viene a suplir el sentimiento de incapacidad de 'estar al día' y constituye, sin lugar a dudas, el motor de la Inteligencia Competitiva (Martinez 2012). La relación entre la vigilancia y la traducción se empieza a vislumbrar cuando la información a la que han de tener acceso hoy en día tanto empresas como organismos públicos no está emitida en una sola lengua, una sola cultura, sino que tiende a ser cada vez más multilingüe, multisoporte y multicultural. En este contexto de manejo de la información no solamente la traducción como actividad sino también el traductor tienen un lugar central. El traductor se convierte, por ende, en un profesional que desarrollará una nueva actividad: la traducciónvigilancia. Así, esta constituye, sin lugar a dudas, una nueva salida profesional para los traductores. Como decíamos al inicio de esta introducción, la traducción como actividad evoluciona de forma extremadamente rápida, de acuerdo con las necesidades del mercado. No se traduce hoy de la misma manera que se hacía hace tan sólo cinco años. La traducción-vigilancia es justamente el resultado de una nueva necesidad del mercado global en el que las empresas u organismos públicos necesitan vigilar un determinado aspecto o adquirir una información concreta emitida en una lengua y cultura que desconocen. El mercado de la traducción es un mercado que goza de plena salud que siguió creciendo incluso durante los años de crisis económica y financiera. En el año 2017 el mercado supuso un volumen de negocios de más de 43 mil millones de dólares con un crecimiento estimado de más del 6\% (Varios autores s/d).

El objeto de este artículo es tratar de definir la traducción-vigilancia como nueva modalidad de traducción y servicio que pueden prestar los traductores profesionales constituyendo sin lugar a dudas un nuevo nicho de mercado. Nuestro objetivo principal consiste en dar los primeros pasos hacia la caracterización de la traducción-vigilancia. En un primer apartado explicaremos qué es la vigilancia y sus implicaciones antes de, en un segundo capítulo, presentar las principales aportaciones existentes en lo referente a la relación entre traducción y vigilancia. Pensamos que las competencias del traductor son compatibles con la prestación de un servicio de traducción-vigilancia. No pretendemos afirmar que el traductor, con su formación en traducción, puede realizar una vigilancia multilingüe, sino que las competencias y aptitudes adquiridas durante su formación universitaria podrían constituir, bajo nuestro punto de vista, el sustrato idóneo para implantar una formación ad hoc en traducción-vigilancia. Por este motivo, en el último apartado presentaremos nuestra propuesta de formación, combinando las subcompetencias (PACTE 2011) adquiridas por los estudiantes de traducción en el Grado y las competencias concretas de la vigilancia estratégica. 


\section{La vigilancia}

La vigilancia consiste en una actividad de seguimiento informativo para detectar tanto amenazas como oportunidades en un determinado sector o ámbito de especialización con el fin de facilitar al cliente o destinatario final una información transformada en conocimiento útil, que este podrá utilizar en la toma de decisión. El papel del vigilante consiste en tratar de limitar el riesgo al facilitar informaciones con un alto valor añadido. La información se transforma en la pieza central del trabajo de vigilancia, la materia prima fundamental que el vigilante tendrá que encontrar, filtrar y tratar.

La vigilancia es a menudo considerada como fundamental en el proceso de definición de la estrategia de una empresa u organización. Su papel es identificar y procesar datos o informaciones útiles que permitirán al destinatario final definir su estrategia y tomar decisiones con conocimiento de causa.

La vigilancia informativa es una actividad humana antigua cuyas referencias más importantes provienen de la aplicación a la esfera civil de prácticas y técnicas empleadas por los servicios de inteligencia. A la hora de emprender una actividad, ya sea empresarial, organizacional o relacional, la necesidad de informarse, de vigilar el entorno y a los competidores, ya sean amigos o enemigos, de compararse con los demás y conocer las tendencias del mercado, es una constante en la historia. La humanidad ha producido en los últimos veinte años más información que en 2000 años de historia y este volumen de información aumenta de forma exponencial cada año. La calidad del filtro es, pues, fundamental.

Este dato, en el que destaca la importancia de una buena criba de la información, es el origen de la necesidad que tienen las empresas de establecer un proceso avanzado y perfeccionado de búsqueda, tratamiento y difusión de informaciones. Desde esta perspectiva, la vigilancia consiste en dar la buena información a la persona correcta en el momento adecuado para tomar la mejor decisión. La gran cantidad de información almacenada en las megabases de datos en todo el planeta permite deducir que hace falta una verdadera metodología para el tratamiento y análisis de la información a causa de la verdadera infoxicación de la que sufrimos. El secreto reside en tratar de luchar contra lo que dice el conocido adagio francés "trop d'info, tue l'info"2.

Numerosos autores y teóricos del campo de la vigilancia proponen, desde su observación de la práctica, dividir la vigilancia en vigilancias más específicas. Estas difieren entre sí esencialmente por el tipo de información manipulada

2. Demasiada información mata la información (traducción propia). 
y los medios puestos en práctica para obtenerla. El método empleado o el proceso involucrado en cada una de estas vigilancias es básicamente idéntico. La principal diferencia consiste en tener en cuenta la heterogeneidad de la información tanto en lo relativo a su formato como a su naturaleza, para adaptar las herramientas utilizadas en la búsqueda, tratamiento y difusión de la misma. A continuación, indicamos una lista de tipos de vigilancia. Esta lista no pretende ser exhaustiva puesto que existen tantos tipos de vigilancia como sectores de actividad y finalidades del cliente:

- La vigilancia científica hace referencia únicamente a los productos y procesos científicos, es decir los artículos publicados en revistas, las tesis, los diferentes informes de investigación, etc.

- La vigilancia tecnológica se basa en la identificación de las evoluciones de las técnicas y tecnologías utilizadas, susceptibles de ser utilizadas por la competencia.

- La vigilancia jurídica y reglamentaria consiste en un seguimiento de las evoluciones de los textos legales como normas y reglamentos que pueden tener una influencia en la oferta de un producto o incluso en la implantación de una empresa en un determinado sector, región, país, organización, etc. Por ejemplo, una multinacional del sector del automóvil que ha sabido anticipar la aprobación de una nueva ley de subvenciones sobre energías renovables podrá lanzar la producción de una nueva línea de vehículos que empleen dicha tecnología.

- La vigilancia comercial se basa en las necesidades de los clientes y los productos lanzados en el mercado. Las necesidades pueden consistir en una solicitud explícita o más tácita. La vigilancia llevada a cabo, por ejemplo, por una entidad bancaria le permite identificar en su clientela una parte importante de clientes que donaban dinero a asociaciones caritativas. El resultado del informe de vigilancia podría permitir a la entidad tomar la decisión de ofrecer a dichos clientes fondos de inversión éticos, con un interés tanto social como económico.

- La vigilancia sociocultural se basa en los comportamientos sociales y factores culturales de los diversos grupos de consumidores de un mercado determinado.

- La vigilancia de imagen (o reputación): se centrará en la percepción desde el exterior de la imagen de una empresa u organización.

- La vigilancia mediática: se refiere al seguimiento de la información publicada en los medios de comunicación.

- La vigilancia sanitaria: tiene un carácter fundamentalmente anticipativo, con la monitorización de nuevos fármacos o focos de enfermedades. 
- La vigilancia de la competencia es sin duda la más practicada, permite comprender la situación actual y la política de los competidores a través del análisis de su situación financiera, los nuevos productos lanzados en el mercado, su investigación en materia de innovación, etc.

Cabe destacar que los distintos tipos de vigilancia pueden estar, y lo están de hecho en la mayoría de los casos, interconectados. De este modo, la vigilancia de la competencia puede incluir una vigilancia tecnológica y estratégica, y así sucesivamente. No existe una separación clara entre los diferentes tipos de vigilancia. Una misma información puede tener un carácter crítico en diversos sectores de vigilancia. Así pues, es necesario que la vigilancia consista en una actividad global. Debe situarse en la intersección entre las diferentes actividades de la empresa para que la información sea explotable en todos los niveles de decisión. No obstante, a nivel procesual, la vigilancia no se lanza de forma multisectorial, es decir que, si bien el proceso de una vigilancia comercial puede llegar a adentrarse en la vigilancia tecnológica, la organización o empresa tiene que tomar una decisión inicial en la definición del proyecto de vigilancia.

Entendemos por vigilancia estratégica toda vigilancia cuyo objetivo final sea la toma de decisión por parte del cliente. El proceso de vigilancia en estos casos generará un producto cuya finalidad será impulsar una decisión tomada a partir del conocimiento útil aportado por el vigilante. El producto fruto de la vigilancia tendrá un carácter estratégico en la medida en que esta tenga una importancia o influencia en la toma de decisión del decisor. Podríamos decir que la gran mayoría (por no decir todas) de las vigilancias están orientadas hacia la toma de decisión, hacia un objetivo claramente estratégico puesto que el producto de la misma siempre será utilizado, en mayor o menor medida, para tomar una decisión, para inclinar la balanza hacia un lado u otro.

Dado que la actividad empresarial sobrepasa a menudo las fronteras, la información que necesitan las empresas es cada vez más multilingüe, multicultural y multisoporte. La limitación de las herramientas de traducción automática y, sobre todo, su incapacidad para 'pensar' o establecer una estrategia de configuración de parámetros de búsqueda y seguimiento informativo multilingüe, hace necesaria la intervención del traductor profesional. La actividad profesional de la traducción-vigilancia es una realidad en el mercado profesional. Son muchas las organizaciones que ya confían en traductores profesionales que han centrado su actividad en la vigilancia multilingüe. Si bien partimos de una observación en la práctica de una realidad nueva, en el apartado siguiente nos centraremos en analizar las principales aportaciones teóricas sobre la relación entre la traducción y la vigilancia. 


\section{Traducción y vigilancia, estado de la cuestión}

El desarrollo cada vez más rápido e importante de las comunicaciones a través de Internet, la multiplicación de los canales de difusión utilizados por los medios de comunicación (ya sean escritos, orales o audiovisuales), la democratización de las herramientas informáticas y multimedia, así como el desarrollo de los medios técnicos, han transformado la información en una materia prima tanto para el sector privado como para el sector público.

El auge de las prácticas de vigilancia formalizada tanto en empresas como en instituciones públicas es un hecho. Vemos cada día ejemplos reales de uso exitoso de la información que nos permiten entender la importancia de obtener información que ofrezca pistas para la toma de decisiones. Si quisiésemos de alguna forma ilustrar la situación en la que se encuentran nuestras sociedades podríamos decir que la 'aldea global' ha incorporado en su seno una gigantesca Torre de Babel que no deja de crecer gracias al desarrollo exponencial de los canales de comunicación. De este modo, la vigilancia del entorno internacional adquiere un lugar preferente en la estrategia de las empresas. La información adquirida y transformada en conocimiento útil constituye una ventaja competitiva que permite facilitar la toma de decisiones. En este sentido, la vigilancia pasa a ser una herramienta fundamental de apoyo a la toma de decisión y adquiere un carácter multilingüe y estratégico.

Podríamos afirmar que el contexto de la globalización de las economías y de las relaciones gracias, principalmente a Internet, está otorgando un papel central a los expertos en comunicación multilingüe, capaces de facilitar la comunicación entre sujetos con culturas y lenguas diferentes (Guidère 2008). La profesión de traductor está en constante evolución debido a la diversificación de los mercados y las posibilidades ofrecidas a través de las nuevas tecnologías y 'autopistas de la información'. La emergencia y, en cierta medida, el uso masivo de nuevas herramientas informáticas tanto de traducción como de tratamiento de texto favorecieron el nacimiento de nuevas ramas o modalidades de traducción (Hurtado 2001), como la localización o la traducción audiovisual, que suscitaron y suscitan cambios profundos tanto en el proceso como en el producto de la tarea traductora.

Hasta donde sabemos, no se ha llevado a cabo hasta la fecha ninguna reflexión en el ámbito hispanohablante (ya sea en España o en países latinoamericanos) acerca de la relación entre traducción y vigilancia estratégica. Por ello, hemos creído oportuno bautizar esta nueva disciplina, inspirándonos de la nomenclatura empleada por Guidère (2008: 118), que combina vigilancia y traducción, conjugando las aptitudes del traductor y las de un experto en 
vigilancia con todo lo que ello implica acerca de las competencias documentales, de búsqueda, tratamiento y análisis, como traducción-vigilancia.

El breve estado de la cuestión que planteamos a continuación pretende ser una visión de conjunto de las aportaciones de los principales autores que han reflexionado sobre la relación entre la vigilancia en sus distintas vertientes y la traducción. El enfoque de esta vista de conjunto de lo que se ha dicho sobre traducción-vigilancia es fundamentalmente práctico y no meramente teórico puesto que los autores presentados son también traductores profesionales. La única bibliografía existente en lo referente a la relación entre vigilancia y traducción la constituyen, por un lado, dos artículos publicados en el año 2007 en la revista Traduire y por otro lado las actas de un coloquio que tuvo lugar en 2008 en la Escuela de Traducción e Interpretación de Ginebra. Bajo nuestro punto de vista, los dos autores más representativos en materia de reflexión teórica en el ámbito de la relación entre la traducción y la vigilancia son Guidère (2008) y Gouadec (2009).

\subsection{La vigilancia multilingüe - Mathieu Guidère (2008)}

Mathieu Guidère es uno de los primeros y pocos autores en haber abordado la cuestión de la emergencia de una nueva modalidad de traducción reflejada en la tarea de vigilancia estratégica. El profesor Guidère es conocido por sus numerosos trabajos relacionados con el análisis del discurso publicitario y político, así como de aspectos socioculturales referidos a grupos terroristas islamistas en Iraq y otros países árabes. Entre los años 2003 y 2007, en su calidad de director del Laboratorio de análisis estratégico y de vigilancia tecnológica (LAISVT por sus siglas en francés) puso en práctica la vigilancia (multilingüe) en un proyecto internacional de vigilancia cuyo objetivo era realizar un seguimiento de los grupos radicales fundamentalistas islámicos, principalmente en las zonas de Oriente Próximo. En su artículo "La veille multilingue: défense et illustration de la traduction stratégique" (Guidère 2008), el autor observa las profundas y constantes evoluciones que experimenta la práctica de la traducción y plantea el aspecto estratégico en el que se enmarca la labor del traductor. En efecto, señala que la traducción es la manera más simple y eficaz de promover la diversidad lingüística y cultural. El traductor, en un contexto de globalización y de intercambios de información en lengua extranjera, se transforma gracias a su papel de mediador intercultural en vigilante multilingüe.

En cuanto a la nomenclatura, Guidère (2007: 47) se refiere a la terminología anglófona y subraya que la actividad de vigilancia en francés tiene un carácter humano y no tan orientado hacia la automatización o la mecanización como en inglés con la utilización de términos como monitoring, scanning, 
screening. Para Guidère, la Inteligencia Competitiva (o inteligencia económica según la traducción literal del francés) se refiere a actividades de espionaje industrial o vigilancia competitiva, haciendo una amalgama y mezclando los conceptos de Inteligencia Competitiva y vigilancia que, como ya hemos explicado en el apartado anterior, poco tienen que ver el uno con el otro a pesar de estar interconectados.

Para el autor, el objetivo de la vigilancia multilingüe es "préparer et étayer constamment la prise de décisions, c'est à dire d'aider les décideurs politiques et économiques à faire les bons choix ou à appuyer les choix stratégiques qu'ils font" Guidère (2008: 18). El aspecto estratégico de la traducción-vigilancia está fundamentalmente ligado a la toma de decisión, al objetivo del producto entregado por el traductor al iniciador de la solicitud de vigilancia. Guidère (2008: 22) distingue cinco etapas distintas en el proceso de traducción-vigilancia: el diagnóstico, la recogida de información, el análisis y finalmente la síntesis de la misma antes de la fase de difusión.

2.2 El traductor-vigilante en el marco de las nuevas profesiones de la traducción - Gouadec (2009)

Gouadec (2009), en su descripción de las distintas modalidades existentes en torno a la profesión de traductor, dentro del apartado dedicado a los oficios de la traducción, menciona aquellas ramas de la traducción que denomina "Traducteur à double compétence". Es decir, traductores cuyas competencias se alían a otras para realizar un trabajo determinado. Entre dichas profesiones encontramos, según Gouadec, el 'traductor de vigilancia'. Se trata aquí de:

Un traducteur chargé de repérer les informations se rapportant à un ou plusieurs sujets particuliers et à les reformuler / synthétiser / réorganiser / résumer en les traduisant Dans la langue des demandeurs d'information. Il cumule donc les fonctions de recherchiste et de traducteur. La veille en question peut être stratégique, commerciale, technologique ou autre. Elle doit permettre au demandeur de trouver les informations utiles et d'en tirer parti le plus rapidement possible (Gouadec 2009: 61).

Gouadec explica, sin entrar en detalle, que el traductor utiliza motores de búsqueda estándar pero también motores de búsqueda dedicados, generalmente diseñados para dar respuesta a una necesidad de información expresada por un cliente determinado. Por otro lado, Gouadec indica que el traductor, dependiendo del volumen de traducción, puede utilizar como herramienta un programa de traducción automática para tratar toda la información. En cuanto al producto, Gouadec plantea que el traductor-vigilante produce fichas de los documentos o webs localizados, resúmenes analíticos y dosieres cada vez más 
completos, traduciendo solamente lo estrictamente necesario y presentando la información útil de la forma más clara y simple posible.

Según Gouadec, nos encontramos frente a una auténtica redefinición del traductor ya que este debe ser capaz de asumir una multitud de funciones y ser tanto un documentalista, buscador de información, técnico, terminólogo, fraseólogo, traductor, adaptador, relector, revisor, etc. El autor presenta una larga lista de las muchas y variadas funciones que puede adoptar un traductor cuyo papel renovado es claramente polifacético y multidisciplinar en el marco de la comunicación multilingüe:

Si elle demeure le moyen privilégié de la communication internationale multilingue, la traduction doit être replacée, aujourd'hui, au centre d'une galaxie. En effet, autour de la traduction se sont développés des métiers qu'il est convenu d'appeler les métiers de la traduction [...]. Que l'on considère ces métiers comme des extensions de la traduction ou qu'on les voie comme de nouveaux métiers, l'important est de savoir qu'ils se développent rapidement et qu'ils tirent de plus en plus les traducteurs vers des activités, des responsabilités, et des compétences d'ingénieur en communication multilingue multimédia (Gouadec 2009: 62).

De este modo, Gouadec identifica la necesidad de situar la traducción y al traductor en un nuevo marco profesional en el que este tiene que adaptarse a las necesidades tanto de mercados como de clientes y desarrollar nuevos perfiles que quizás se alejen de las definiciones tradicionales de traductor. En su Guide des métiers de la traduction-localisation et de la communication multilingue et multimédia Gouadec (2009) plantea la relación entre traducción y vigilancia, pero asimila dicha actividad a la de terminología, otorgando al traductor-vigilante las mismas competencias que las del terminólogo:

En toute logique, le terminologue est un "veilleur". Il l'est doublement, il assure d'abord tout naturellement une veille terminologique permanente au sens où il repère, recense, intègre et traite les nouveaux termes qui apparaissent dans son horizon professionnel et dans les domaines d'activité des commanditaires de son travail. Mais il est également chargé d'assurer la veille technologique ou commerciale ou stratégique pour le compte de divers servies ou clients commanditaires. [...] Le terminologue est le veilleur idéal parce qu'il est un véritable spécialiste de la documentation, d'une part (il est par nature un fureteur efficace et ses stratégies de recherche d'informations sont, en principe, d'une efficacité redoutable) et parce qu'il maîtrise des langues étrangères, d'autre part (Gouadec 2009: 32).

En lo referente a la traducción-vigilancia propiamente dicha, el autor define de la manera siguiente las tareas y el cometido del traductor-vigilante:

Les traducteurs de veille représentent une catégorie particulière de traducteurs qui traquent l'information commerciale, stratégique, technologique ou autre, 
souhaitée par leurs clients. Ils recherchent les sources d'information (sur le web), sélectionnent l'information pertinente, la traduisent, la remettent en forme et la rediffusent généralement par voie de lettre d'information ou via un intranet ou extranet protégé (Gouadec 2009: 68).

\subsection{La traducción-vigilancia, una nueva salida profesional para los traductores}

Gracias a la emergencia y desarrollo cada vez más rápido de las ciencias de la información, la función comunicativa de la traducción cobra una importancia innegable. En dicho contexto, la traducción no constituye una función como cualquier otra de la comunicación interlingüística y el traductor ya no representa un mero enlace entre el emisor y el receptor, entre dos textos. Más allá del soporte textual, la traducción se sitúa en un contexto de mediación lingüística, pero a su vez cultural y social. Guidère (2008: 16) plantea que la traducción se transforma en comunicación multilingüe por constituir una mediación con valor social que se desarrolla a través de un proceso en tres etapas: la identificación del destinatario, la definición del lenguaje específico y el establecimiento de una relación basada en los conocimientos comunes. La labor del traductor en este contexto va más allá de sus tareas «tradicionales», se transforma en actor estratégico debido a las decisiones que tiene que tomar, con el fin de identificar adecuadamente el tipo de comunicación para optimizar el proceso de comunicación multilingüe.

La traducción-vigilancia, entendida como una actividad de seguimiento informativo en dos o más idiomas sobre una temática definida por el iniciador del encargo, es una actividad relativamente reciente debido a la juventud de la disciplina más amplia en la que se engloba, la Inteligencia Competitiva (Martinez 2012). La aplicación de un sistema de vigilancia formalizada en el seno de una estrategia empresarial o estatal (en este caso dejando de lado las prácticas de los servicios de inteligencia que no son objeto del presente estudio) es relativamente reciente, más aún si nos situamos en el contexto español. Asimismo, al igual que hemos encontrado poca bibliografía en español en lo referente al campo general de la vigilancia estratégica e Inteligencia Competitiva (la mayoría de la bibliografía acerca de estas dos disciplinas la hemos encontrado en lengua inglesa y francesa), la bibliografía en lo referido a las implicaciones de la vigilancia en materia de traducción es muy escasa. Tal y como planteábamos, el trabajo pionero que estableció una relación entre la vigilancia estratégica, la traducción y la traductología fue un artículo publicado en la revista Traduire en 2007 por el profesor Mathieu Guidère y, posteriormente, los artículos de las actas del coloquio que tuvo lugar el 28 y 29 de mayo de 2008, organizado por la Escuela de Traducción e Interpretación de Ginebra (ETI Suiza) y por el GRETI (Grupo 
de Investigación en Traducción e Interpretación) cuyo objetivo fue el estudio de la relación entre la traducción y la vigilancia estratégica en el marco actual de globalización de la economía y generalización de las herramientas de comunicación en todas las lenguas. Por otro lado, cabe mencionar la obra del profesor Gouadec del año 2009 Guide des métiers de la traduction en la que menciona la actividad de traducción-vigilancia como una de las nuevas profesiones de la traducción surgidas a raíz de las necesidades de información de las empresas y en la que el traductor es un actor cuyas competencias son las más adecuadas para realizar dicha labor.

Podríamos decir que el coloquio que tuvo lugar en Suiza en el año 2008 fue precursor en materia de vigilancia multilingüe (traducción literal de la terminología empleada por la mayoría de los autores que hemos analizado) y que el estudio sobre el fenómeno se encuentra en ciernes puesto que carece de un marco teórico al no haberse llevado a cabo hasta la fecha investigaciones de envergadura que permitan corroborar o refutar posibles hipótesis acerca de las orientaciones de esta nueva salida para los traductores profesionales.

El nexo de unión o el punto común de la gran mayoría de los autores cuyas reflexiones hemos mencionado es el lado eminentemente práctico de sus planteamientos. En efecto, todos los autores, además de ser en algunos casos docentes de la traducción, son profesionales que ponen en práctica en su día a día la traducción-vigilancia. De este modo, podemos afirmar que la reflexión realizada acerca de esta disciplina, esta nueva rama de la traducción, se basa en la observación del fenómeno.

Consideramos que Guidère es uno de los autores que hace más hincapié en el aspecto teórico de la disciplina ya que realiza una reflexión acerca de las implicaciones de la vigilancia para el traductor y la profesión de la traducción. Para él, el traductor adquiere un papel estratégico ya que su intervención es fundamental para la realización de la vigilancia gracias a sus competencias tanto lingüísticas, culturales como analíticas. No obstante, Guidère realiza una amalgama entre Inteligencia Competitiva y espionaje, distinguiéndolos de la vigilancia cuando en realidad, son realidades imbricadas. En efecto, la Inteligencia Competitiva y la vigilancia nada tienen que ver con el espionaje industrial ya que se realizan partiendo de información blanca o abierta y no negra o ilegal. Por otro lado, consideramos que la vigilancia forma parte de la Inteligencia Competitiva, disciplina madre que de alguna forma la engloba. De este modo, no se puede confundir Inteligencia Competitiva con vigilancia. Los planteamientos presentados por Guidère nos parecen de sumo interés ya que de alguna manera el autor pone las primeras piedras de lo que podría ser un enfoque traductológico para la traducción-vigilancia. 
Una de las últimas aportaciones en materia de vigilancia multilingüe es la que plantea Gouadec, aunque sea de forma muy breve, en uno de sus últimos libros Les métiers de la traduction (Gouadec 2009). La aportación de Gouadec nos parece muy interesante ya que confirma que la vigilancia se está transformando en uno de los nuevos campos en los que el traductor tiene un lugar fundamental. En cuanto a la metodología, el autor indica que el traductor traduce lo estrictamente necesario presentando una información clara, precisa y simple. Para Gouadec, el traductor es un actor multifunciones, motivo por el cual la vigilancia se transforma en una de las esferas de acción para éste. La secuencia metodológica presentada por Gouadec nos parece interesante a pesar de que, bajo nuestro punto de vista, carece de fundamento teórico o práctico ya que el autor no ilustra con ejemplos la metodología que presenta. Por otro lado, el autor asimila al traductor-vigilante con el terminólogo al considerar que las competencias necesarias para la realización de la vigilancia son las mismas que las que emplearía un terminólogo en el desempeño de sus labores. No consideramos que el traductor-vigilante pueda asimilarse con un terminólogo puesto que, si bien ambas actividades tienen un componente textual y conceptual, la traducción-vigilancia va mucho más allá de la terminología, sobre todo en el ámbito analítico. El traductor no solamente tiene que elegir de forma correcta las palabras clave de su búsqueda, sino que tiene que ser capaz de seleccionar, analizar, condensar y traducir la información hallada para ofrecer al cliente o destinatario del servicio de vigilancia un producto cuyo objetivo es favorecer la toma de decisión. Como menciona Gouadec, el traductor-vigilante se caracteriza por su faceta multidisciplinar, pero añadiremos también por su capacidad de trabajo en red. En efecto, el esquema de trabajo de la traducción-vigilancia implica una colaboración estrecha entre todos los actores del proceso con el fin de generar un producto de calidad que sirva su función primera: ser una herramienta para la toma de decisiones.

Al igual que ocurre en otras modalidades de traducción, pensamos que la traducción-vigilancia, una vez identificada como nueva disciplina, requiere una formación específica orientada hacia la adaptación y reorientación de aquellas aptitudes y subcompetencias adquiridas por los traductores en formación o traductores profesionales que siguen formándose. Como decíamos en la introducción, partimos de una constatación u observación de una realidad del mercado profesional en el que ha aparecido un nuevo tipo de demanda de servicios a la que el traductor puede aportar soluciones dado que la traducción automática no puede aportarlas al requerirse una profunda capacidad de reflexión y de análisis. Así las cosas, el perfil del traductor parece ser adecuado 
para la prestación de un servicio de vigilancia multilingüe, no obstante, dada la novedad de esta disciplina, dicho perfil requiere, como es lógico, una formación específica que, bajo nuestro punto de vista, podría consistir en complementar la formación universitaria existente. Las conversaciones con actores y empresas del sector de la vigilancia (monolingüe, es decir realizada en una sola lengua) nos han indicado y demostrado que existe una necesidad de resolver el 'problema' multilingüe existente en la vigilancia y que las herramientas de traducción automática no son una solución viable, al menos en las etapas iniciales del proceso. Basándonos en la observación de nuestra propia experiencia profesional, así como en las características de las asignaturas existentes en el Grado de traducción e interpretación así como las competencias necesarias para la realización de la vigilancia estratégica (Varios autores 2007), presentamos a continuación nuestra propuesta provisional de formación en traducción-vigilancia.

\section{Hacia una propuesta didáctica provisional}

Tanto nuestra experiencia profesional como el breve estado de la cuestión llevado a cabo en el presente artículo sobre la relación entre la traducción y la vigilancia nos llevan a afirmar que parece existir una nueva salida profesional para los traductores. La vertiente multilingüe y multicultural de la vigilancia es una realidad en el mercado. El traductor y la traducción como actividad tienen un lugar central en el proceso de vigilancia multilingüe. Pensamos que el perfil del traductor podría adecuarse a la prestación del servicio de vigilancia siempre y cuando reciba una formación que le permita adquirir las competencias de vigilancia necesarias.

El proceso de traducción-vigilancia implica la manifestación de la mayoría de las subcompetencias de traducción establecidas por PACTE (2003). No obstante, dichas subcompetencias no son suficientes para la correcta realización de la vigilancia multilingüe puesto que están orientadas exclusivamente a la actividad traductora, sin tener en cuenta las necesidades de un cliente que no solicita la traducción de un documento de una lengua a otra sino la monitorización, selección y obtención de información emitida en una o varias lenguas desconocidas para él. En muchos casos, el cliente podría incluso no indicar las lenguas en las que está emitida la información o tampoco concretar qué información necesita realmente. Como consecuencia de ello, la tarea del traductor-vigilante no se limita a realizar un encargo encomendado sin más por un cliente (como es el caso en un encargo de traducción), sino que, en ocasiones, también tiene que orientar al cliente hacia el producto final que 
necesita: qué información buscar, en qué zonas geográficas, qué elementos vigilar, etc. Una vez establecidos todos los parámetros del encargo de vigilancia, el traductor-vigilante tiene que producir un documento de llegada, un informe de vigilancia multilingüe, en la lengua del cliente basado en la información hallada, tratada, analizada y, sobre todo, traducida.

La observación del sector de la traducción y de nuestra propia experiencia profesional como traductor y gerente de agencia de traducción nos ha permitido llegar a la conclusión de que la formación recibida en el Grado de traducción no es suficiente para la realización de la vigilancia multilingüe. Existe una serie de carencias que podrían subsanarse con la implementación de una formación ad hoc en traducción-vigilancia que permitiera combinar la utilización y adaptación de los subcomponentes de la competencia traductora con las competencias necesarias para la realización de una vigilancia.

La necesidad de una formación en traducción-vigilancia se sustenta en el hecho de que parece existir una correlación entre las competencias necesarias para la práctica de la vigilancia multilingüe y el perfil del traductor. En efecto, si bien el traductor parece presentar una serie de competencias adecuadas para la realización de la vigilancia multilingüe, al no haber recibido ninguna formación en lo referente a la prestación de un servicio de ese tipo y que poco tiene que ver con un servicio de traducción per se, necesita recibir una formación específica que le permita adaptar ciertas capacidades ya adquiridas y adquirir otras que aún desconoce. Somos conscientes de que basamos nuestra propuesta en la mera observación de la práctica de una nueva realidad en el mercado profesional y que la puesta en práctica es la que permitiría corroborar o refutar nuestra hipótesis.

Dada la juventud de esta nueva modalidad o tipo de servicio de traducción, hasta la fecha, no se ha realizado ningún estudio que permita determinar un punto de partida de una posible formación en traducción-vigilancia. Si nos basamos en el análisis del plan de estudio y de las guías docentes del Grado en Traducción e Interpretación de la Universidad de Alicante, podríamos decir que las asignaturas que ofrecen más pistas acerca de la realización de una vigilancia multilingüe son las asignaturas de traducción técnico-científica y las de terminología I y II. Si bien es verdad que ambas asignaturas presentan elementos formativos que parecen sentar las bases de las aptitudes y competencias necesarias para dar los primeros pasos en una vigilancia informativa multilingüe, dichos elementos formativos están, como es lógico, exclusivamente orientados a la actividad traductora (englobando las distintas modalidades de traducción, así como las actividades que gravitan alrededor de la misma: redacción, revisión, localización, etc.). Uno de los aspectos en los que se basa la docencia de 
las dos asignaturas mencionadas es la capacidad de los alumnos para documentarse, encontrar y generar bases de datos terminológicas, en el caso de la asignatura de terminología; y, en el caso de la traducción técnico-científica, de ser capaces de encontrar todos los recursos necesarios que puedan ayudarles para la realización de la traducción, resolver problemas terminológicos, de comprensión, de formato, etc. Encontramos justamente en lo que acabamos de mencionar uno de los motivos por los cuales el estudiante de traducción es el candidato idóneo para una formación en traducción-vigilancia puesto que ya ha adquirido una serie de aptitudes y competencias necesarias para la correcta realización de la vigilancia multilingüe, sin ser consciente de ello. No obstante, dado que poco tiene que ver la actividad de traducción en sí con la de traducción-vigilancia, existe una necesidad patente de adaptar dichas aptitudes, subcompetencias de traducción o de forma más general, aspectos formativos, a la realización de la traducción-vigilancia.

Dado que no existe en la actualidad ninguna formación oficial, universitaria, en materia de traducción-vigilancia, se podría diseñar una formación ad hoc bajo la forma de una asignatura de nivel Máster que se centrara exclusivamente en la traducción-vigilancia, combinando la adaptación de competencias ya adquiridas y la implantación de elementos formativos no adquiridos y que resultan necesarios. La elección del nivel Máster para la implantación de esta formación está motivada por el nivel de pericia traductológica adquirida por los estudiantes, una vez completados los estudios de Grado.

Nuestro planteamiento de estrategia didáctica para la formación en traducción-vigilancia parte desde la observación de la realidad profesional pero también del estudio de casos. Hemos dividido nuestro plan de formación en tres grandes bloques con el fin de que el discente pueda adentrarse en el proceso y entender sus engranajes. Nuestro objetivo es trasladar a la formación los distintos pasos del proceso. A continuación, detallamos los tres bloques que constituyen el contenido de la asignatura de traducción-vigilancia:

1) Introducción

2) Definición de las necesidades y comprensión del encargo

3) Búsqueda de fuentes y de información, y recogida de información

4) Análisis y tratamiento de la información, generación y difusión del informe 
Contenidos de la asignatura:

Bloque 0 - Introducción

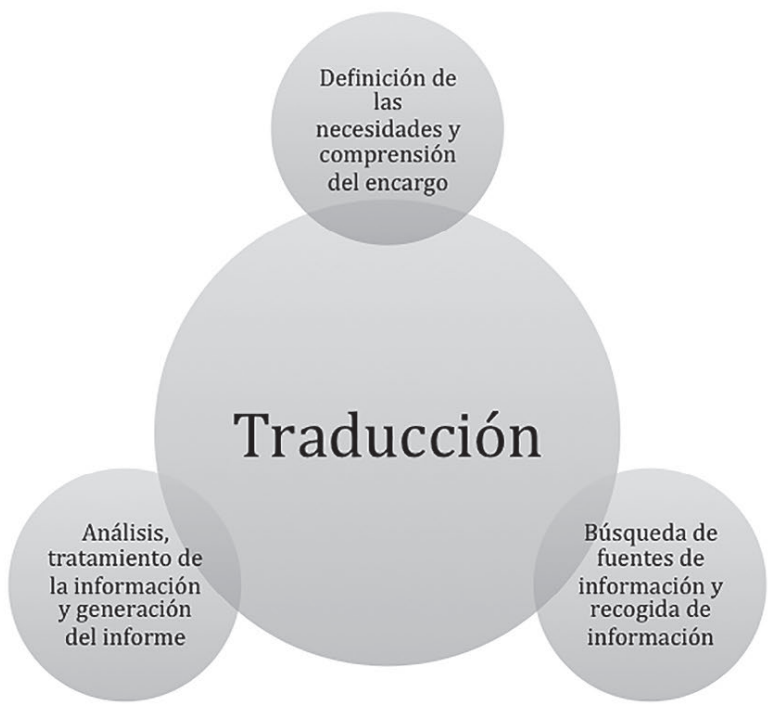

Introducción:

- Qué es la vigilancia.

- Qué es la Inteligencia Competitiva y su relación con la vigilancia.

- Modelos, fuentes y sistemas.

- Cadena de valor de la información.

- Presentación del ciclo de la vigilancia.

- Vigilancia y multilingüismo/multiculturalismo.

- Vinculación entre la traducción y la vigilancia multilingüe.

- Definición del proceso de vigilancia multilingüe (explicación del flujograma). 
Bloque 1: Definición de las necesidades del encargo de vigilancia

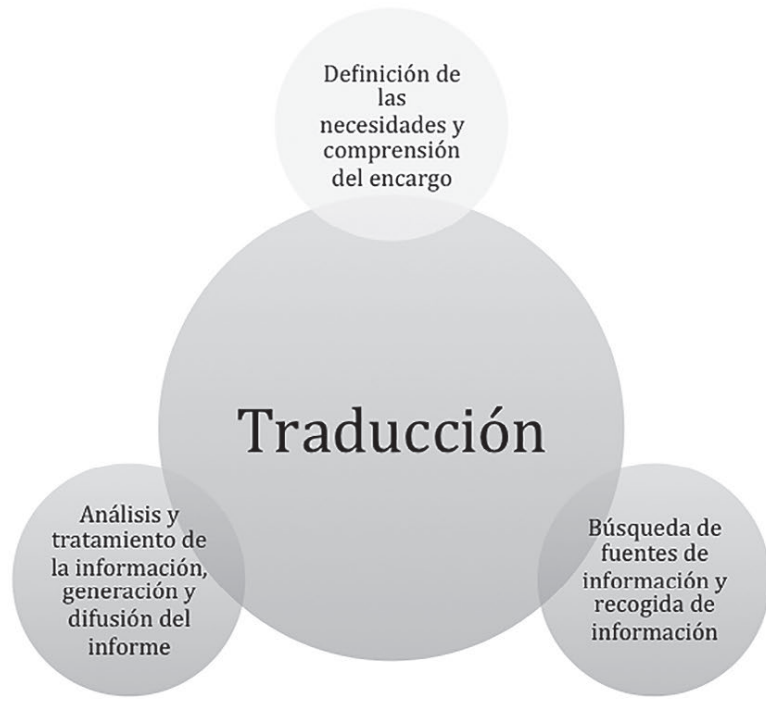

Bloque 1.1 Exploración inicial: descubrimiento de temática, conceptos, agentes y palabras clave.

- Buscadores y metabuscadores (Google como herramienta profesional).

- Alternativas a metabuscadores.

- Definición y configuración de una ecuación de vigilancia.

- Definición y traducción de palabras clave.

- Ecuación de búsqueda, vigilancia y traducción: principales implicaciones.

Bloque 1.2 Configuración de las primeras vigilancias y alertas: puesta a prueba de palabras claves, descubrimiento de fuentes, recepción de primeras informaciones.

- Utilización de herramientas de: agentes inteligentes, rastreadores de noticias, RSS.

- Retroalimentación del proceso en los primeros pasos: búsquedas iniciales y hallazgos reorientan primeras búsquedas. 
Bloque 2: Búsqueda de fuentes de información/búsqueda de información y recogida de información

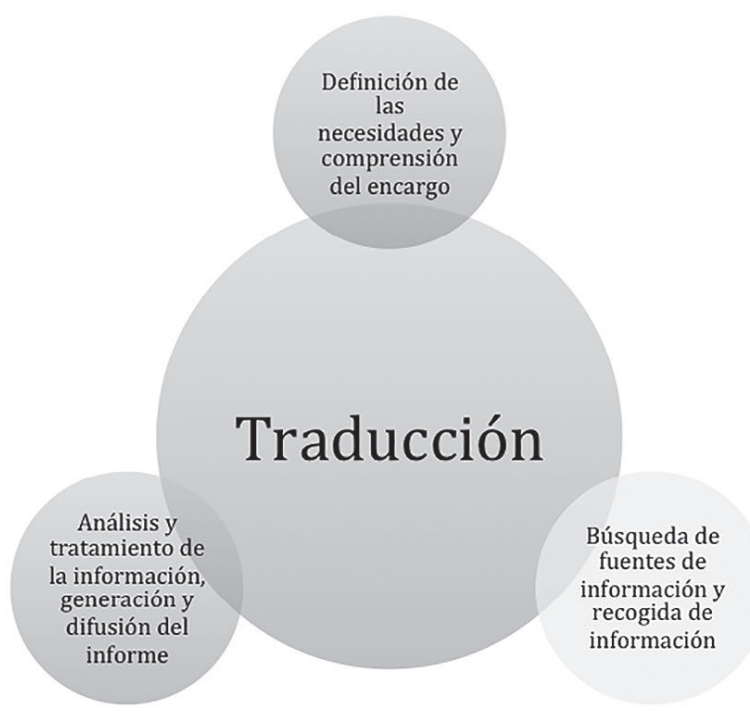

Bloque 2.1 Definición de los distintos tipos de fuentes de información

- Fuentes generales

- Fuentes técnicas

- Fuentes secundarias

- Informadores

- Web invisible

- Criterios de determinación de la fiabilidad de las fuentes de información

Bloque 2.2 Asentamiento de la búsqueda de información: aplicación de las ecuaciones de búsqueda con recogida de información

- Detección de nuevos contenidos

- Recopilación/recogida de información y organización de la misma para posterior tratamiento.

- Uso de herramientas de vigilancia: recopiladores de información, hilos RSS

- Aplicación de la minería de datos a la vigilancia multilingüe.

- Gestión del conocimiento: uso de plataformas de gestión del conocimiento antes del tratamiento.

- Indexación de la información 
Bloque 3: Análisis y tratamiento de la información, generación y difusión del informe de vigilancia multilingüe

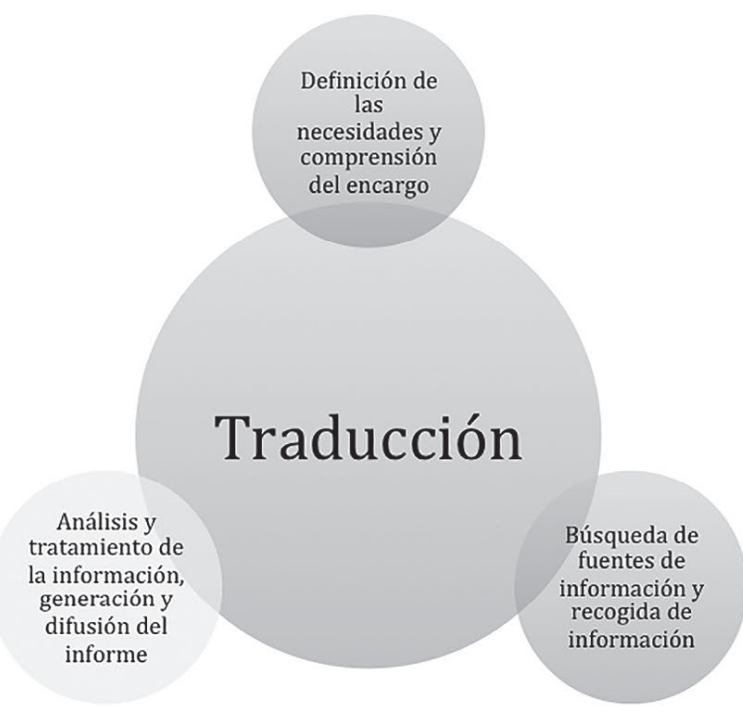

Bloque 3.1 Traducción-vigilancia, instrumentalización de la traducción para la generación de conocimiento útil:

- Las distintas estrategias de traducción a la hora de generar el informe de vigilancia.

- La traducción selectiva.

- La traducción resumida.

- La traducción completa.

Bloque 3.2 Herramientas de tratamiento de la información: programas de minería de datos multilingüe.

- Bloque 3.3 Elaboración del informe de vigilancia multilingüe.

- Bloque 3.4 Métodos de difusión del producto final: diversos soportes para diversas necesidades informativas. Informe de vigilancia transmitido a través de:

- Email, newsletter.

- Blog.

- Documento pdf con enlaces.

- Notas de alerta.

Bloque 3.5 Aspectos deontológicos y éticos de la traducción-vigilancia. 


\section{A modo de conclusión}

La multiplicación de las comunicaciones, el desarrollo exponencial de la cantidad de información publicada en internet, la velocidad vertiginosa de difusión de dicha información y la interacción cada vez más importante entre culturas y lenguas, constituyen factores que otorgan necesariamente un carácter multilingüe a la vigilancia. La relación entre la vigilancia y la traducción parece surgir de forma natural en el momento en el que un cliente solicita una vigilancia que tiene que versar sobre una determinada temática que requiera el acceso a una información multilingüe y multicultural. El traductor ya no se limita a traducir un texto de una lengua $A$ a $B$, sino que tiene que producir en la lengua del cliente un documento que le permita obtener información sobre una temática concreta para la toma de decisión del destinatario final. Así pues, teniendo en cuenta el vacío teórico, la novedad de la disciplina y la ausencia de tratamiento en lengua española, decidimos denominar la actividad combinada de traducción y vigilancia 'traducción-vigilancia', siendo el actor principal de la misma el 'traductor-vigilante'.

La traducción-vigilancia es una actividad novedosa dentro del campo de la traducción y representa una salida profesional emergente llena de posibilidades para los traductores tanto profesionales como en formación. Esta afirmación fue el motor de arranque de nuestra investigación, así como nuestra primera hipótesis de partida gracias a la constatación, fundamentada en la observación de nuestra propia experiencia profesional, de una realidad del mercado de la traducción que no parecía haber tenido tratamiento en el ámbito de la traductología moderna. La novedad de lo que hemos venido calificando como fenómeno, disciplina o modalidad, conllevaba una acuciante necesidad de caracterización, definición y puesta en perspectiva tanto para los traductores profesionales como para los traductólogos. Como conclusión de los diferentes apartados del presente artículo podríamos decir que:

1- La traducción-vigilancia constituye efectivamente una nueva salida profesional para el traductor. La traducción constituye un elemento central de la comunicación y de los intercambios de bienes y servicios en el seno del fenómeno de globalización galopante en el que se encuentran nuestras sociedades. No obstante, detectamos que el mercado ha generado un nuevo tipo de solicitud de servicios que no se centra en la mera solicitud de traducción de un texto redactado en una lengua $\mathrm{A}$ hacia otra lengua $\mathrm{B}$, sino que aquí el cliente, ya sea particular, del sector privado o público, solicita una información a la que no tiene acceso al haber sido emitida en una cultura y lengua que desconoce. La traducción-vigilancia surge como la actividad de búsqueda y seguimiento 
informativo en una o más lenguas extranjeras para la transformación de la información encontrada en conocimiento útil en la lengua del cliente para la toma de decisión.

2- La traducción-vigilancia otorga al traductor un nuevo papel en el seno de la comunicación multilingüe. Al parecer, el papel del traductor cambia a la hora de llevar a cabo un proyecto de traducción-vigilancia. Cuando el traductor tiene que realizar una traducción encargada por un cliente, no tiene poder de decisión sobre el documento que tiene que traducir y en muchos casos no sabe para qué va a servir su traducción. Podríamos decir que su tarea se limita a traducir de una lengua a otra un documento. La diferencia con la traducciónvigilancia reside en el hecho de que el encargo no es un documento que ha de ser traducido sino más bien una solicitud de información emitida en lengua extranjera. El traductor tiene que tomar decisiones en cuanto a la búsqueda, la selección de información, las estrategias de vigilancia, la periodicidad, etc. Además, debe decidir también qué fragmentos o textos completos tiene que traducir, qué tipo de traducción tiene que realizar (una traducción más literal o, por el contrario, más adaptada y libre), qué tipo de soporte puede consultar para recabar información (video, audio, texto, etc.) y, finalmente, de qué forma se lo va a transmitir al cliente. De cara al tratamiento de un encargo de traducción-vigilancia el traductor tendrá, como consecuencia, un nuevo papel si lo comparamos con el papel del traductor a la hora de prestar un servicio de traducción convencional. Aquí el traductor se transforma en un actor central y fundamental para la toma de decisión del destinatario del producto final.

3- Nuestra propuesta de modelo didáctico, basado en el cruce de competencias de traducción y de vigilancia podría constituir una base para el desarrollo de una formación en traducción-vigilancia. La traducción-vigilancia consiste en un seguimiento informativo en lenguas extranjeras, pero también en culturas diferentes. El traductor, con su formación y las competencias adquiridas durante los estudios de traducción, tiene muchas de las aptitudes necesarias para la realización de la vigilancia en varios idiomas. El modelo de formación que planteamos, basándonos en la praxis de esta nueva disciplina pero también las subcompetencias de traducción y las competencias de vigilancia, podría constituir una antesala para la formación de traductores en traducción-vigilancia.

La traducción-vigilancia plantea una redefinición de la relación entre el traductor y el cliente o destinatario final del producto entregado. Tal y como planteábamos anteriormente, la solicitud de vigilancia multilingüe consiste en una solicitud de información. El cliente no es un cliente que quiere una simple traducción, es decir un cliente que solicita a un traductor la traducción 
de un documento escrito en lengua A para que este lo traduzca y produzca un documento en lengua B. Pensamos que este hecho es esencial en la caracterización de la disciplina, puesto que el encargo no lo constituye un texto en una determinada lengua sino más bien una necesidad informativa, más o menos precisa, sobre una temática concreta expresada en la lengua del cliente. A partir del momento de recepción del encargo el traductor tendrá, en primer lugar, que ser capaz de analizar la necesidad informativa del cliente y establecer exactamente sus necesidades en la o las lenguas de destino. De este modo, el cliente en muchas ocasiones puede solicitar una vigilancia multilingüe sobre, por ejemplo, un competidor en otro país, pero no especificará una limitación lingüística, dejando rienda suelta al traductor-vigilante para que valore la necesidad de ampliar el ámbito de vigilancia hacia otras lenguas o incluso otros campos y ámbitos. Si bien en el marco de un encargo de traducción el traductor puede no conocer o tratar directamente con el destinatario final del documento entregado, en el marco de la traducción-vigilancia dicho contacto es fundamental ya que de él dependerá la correcta comprensión y orientación de la estrategia de vigilancia multilingüe planteada. Por otro lado, en la mayoría de los casos, el producto de traducción-vigilancia tendrá un carácter confidencial al contener información con un alto valor añadido para su destinatario. En lo referente al producto de traducción-vigilancia hablamos de informe de vigilancia multilingüe. Dicho informe podrá haberse elaborado a través del uso de distintas estrategias de traducción: la traducción completa, selectiva o resumida. La traducción-vigilancia se concibe como un proceso cíclico en el que el traductor-vigilante tiene que acudir de forma regular al cliente para presentarle resultados que pueden reorientar todo el ciclo debido al hallazgo de una información relevante y que implique una nueva necesidad informativa.

Pensamos que la traducción-vigilancia constituye un campo de estudio con muchas posibilidades tanto para los traductólogos como para los traductores profesionales. Así las cosas, una de las vías futuras de investigación que se podría plantear es la de seguir avanzando hacia una caracterización del perfil del traductor-vigilante a través de un análisis más profundo del proceso y del producto. Pensamos que el producto de la traducción-vigilancia, entendido como un producto de traducción dinámico, podría analizarse de forma más profunda con el fin de definir estrategias concretas de traducción. Por otro lado, sería interesante analizar la toma de decisión del traductor en este último aspecto. Ello nos permitiría, tal vez, sistematizar la enseñanza de dichas estrategias. La utilización de programas de eye tracking nos permitiría analizar de forma concreta el proceso seguido por el traductor-vigilante a la hora 
de seleccionar la información relevante y pertinente en el marco del encargo planteado por el cliente.

Finalmente, nos parece fundamental mencionar un aspecto que no hemos tratado en el presente artículo y que plantea un horizonte totalmente nuevo para el traductor: las implicaciones del traductor en la toma de decisión del cliente o lo que es lo mismo, los aspectos éticos y deontológicos ligados a la traducción-vigilancia. En efecto, la información se ha transformado en la materia prima del funcionamiento de nuestras sociedades, y en el mundo globalizado en el que vivimos, el traductor-vigilante pasa a ser un proveedor de información multilingüe con alto valor añadido. De este modo, podrían darse casos en los que la información aportada por el traductor, siempre hallada y tratada de forma legal, tuviera consecuencias poco éticas a la hora de su utilización por el destinatario final. Pensamos que la juventud de la disciplina implica un trabajo de reflexión acerca del papel del traductor en la comunicación multilingüe ya que este, tal y como hemos tratado de demostrar a lo largo de este artículo, está ocupando un lugar cada vez más preponderante en la sociedad de la información.

\section{Referencias bibliográficas}

BAKER, Mona. (2006) Translation and conflict. Londres \& Nueva York: Routledge. FRANJIÉ, Lynne. (2008) "Les traducteurs et la veille média: méthodes et exemples." En: Gudière, Mathieu (ed.) 2008. Traduction et Veille stratégique multilingüe. París: Le Manuscrit, pp. 51-77.

GouADEC, Daniel. (2009) Profession Traducteur. París: La Maison du dictionnaire.

GOUADEC, Daniel. (2009) Guide des métiers de la traduction-localisation et de la communication multilingue et multimédia. París: La maison du dictionnaire.

GUIDĖRE, Mathieu. (2007) "Le traducteur-veilleur ou traduction et veille stratégique multilingüe." Traduire 215, pp. 44-62.

GUIDĖRE, Mathieu. (2008) Traduction et Veille stratégique multilingue (coll.). París: Le Manuscrit.

HuRTADO, Amparo. (2011) Traducción y traductología: Introducción a la traductología. Madrid: Cátedra.

MARTINEZ, Patrick. (2012) La traducción-vigilancia: aproximación a una nueva salida profesional para traductores. Alicante: Universidad de Alicante. Tesis doctoral inédita.

Moreno, Guadalupe. (2018) “iQué sucede en un minuto en Internet?" <https:// es.statista.com/grafico/13159/que-sucede-en-un-minuto-en-internet/>

PACTE. (2001) "La competencia traductora y su adquisición." Quaderns. Revista de traducción 6, pp. 39-45. 
VARIOS AUTORES (ADBS). (2007) Référentiel des métiers et des fonctions de l'information-documentation. París: ADBS éditions. Versión electrónica: <https://f-origin. hypotheses.org/wp-content/blogs.dir/12/files/2009/04/referentiel-metier-rm. pdf>

VARIOS AUTORES (Common Sense Advisory). (s/d) "Página de inicio." <http://www. commonsenseadvisory.com/>

\section{NOTA BIOGRÁFICA / BIONOTE}

PATRICK MARTINEZ es licenciado en Traducción e Interpretación por la Universidad de Alicante (2001), posee un Máster en Relaciones Internacionales por la Universidad Complutense de Madrid (2005) y defendió su tesis doctoral en Traducción e Interpretación en la Universidad de Alicante en 2012 obteniendo el título de Doctor en Traducción e Interpretación. Su investigación se centra en la vigilancia estratégica multilingüe. En 2001 empezó a trabajar como traductor e intérprete profesional. En 2004 fundó Tradulingua, agencia de traducción especializada en la comunicación multilingüe a nivel global y en la organización de cursos de idiomas y formación en el extranjero. Patrick Martinez es profesor del Departamento de Traducción e Interpretación de la Universidad de Alicante desde el año 2008; e imparte las asignaturas de traducción técnico científica, interpretación de conferencia en el Grado de Traducción e Interpretación, así como la asignatura de traducción para la propiedad intelectual en el Máster Oficial de traducción institucional.

PATRICK MARTINEZ holds a BA in Translation and Interpretation from the University of Alicante (2001), a Master's Degree in International Relations from the Universidad Complutense de Madrid (2005) and a PhD in Translation and Interpretation from the University of Alicante (2012). His research focuses in Multilingual Strategic Watch. In 2001 he started working as a professional translator and interpreter. In 2004 he founded Tradulingua, a translation agency specialized in multilingual global communication. Dr. Martinez is an Associate Professor of the Translation and Interpretation Department of the University of Alicante since 2008. He teaches technical translation and conference interpretation at both undergraduate and postgraduate level. 
